\title{
PEI - a potent, but not harmless, mucosal immuno-stimulator of mixed T-helper cell response and FasL-mediated cell death in mice
}

\author{
K Regnström, EGE Ragnarsson, M Köping-Höggård, E Torstensson, H Nyblom and P Artursson \\ Department of Pharmacy, Uppsala University, Uppsala, Sweden
}

\begin{abstract}
Polyethyleneimine (PEI) is one of the most effective gene delivery systems available today. However, very little is known about its ability to stimulate a systemic immune response and the molecular mechanisms thereof. However, this information is vital for the future development of new gene delivery systems.

Here we address this issue by studying gene expression profiles from spleen lymphocytes after in vivo immunization of mice with PEI formulated with a reporter plasmid (PEI+) or the formulation alone (PEI-). PEI- was found to provoke the activation of genes with important immunostimulatory functions, but without the necessary costimulatory signals. PEl+ resulted in: a mixed Th1/Th2 response; activation of both
\end{abstract}

$C D 8^{+}$and $C D 4^{+} T$ cells, with a larger effect on $C D 4^{+}$; and FasL-mediated antigen-induced cell death. A comparison of the immune responses of PEI+ with that of the clinically used tetanus toxoid-aluminum phosphate vaccine showed that the DNA vaccine provoked a stronger immune response as compared to the protein vaccine. However, many genes involved in other cellular responses such as apoptosis, stress responses and oncogenesis were activated in PEI+, supporting the theory of immunostimulation by danger genes, but also pointing toward possible adverse reactions such as Alzheimer's disease.

Gene Therapy (2003) 10, 1575-1583. doi:10.1038/

sj.gt.3302054

Keywords: nonviral gene delivery; DNA vaccine; polyethyleneimine; systemic immune response; gene expression; cDNA array

\section{Introduction}

The use of DNA vaccines has been ascribed a promising future, revolutionizing medical technology, and several such vaccines are currently in clinical trials. ${ }^{1}$ Most of these trials concern vaccines based on viral delivery of DNA; however, nonviral delivery systems have recently become more popular.

Naked antigen encoding DNA are known to be able to synthesize the encoded protein and evoke a subsequent immune response of both the cellular and humoral kind, if administered by the intramuscular or intradermal routes. $^{2,3}$ Intranasally administered naked DNA has been shown to evoke a widespread mucosal immunity in animals. ${ }^{4}$

Humoral and cellular immune responses have also been reported for delivery systems based on cationic lipids $^{5}$ and cationic microparticles ${ }^{6}$ and a protective immune response has been observed with orally given Chitosan-DNA complexes in a murine peanut allergy model. ${ }^{7}$

Several other nonviral gene delivery systems have been developed hitherto, but unsolved problems remain. They include targeting to specific tissues if not administered directly to the desired tissue and improvements in all aspects of gene delivery to increase efficiency. Polyethyleneimine (PEI) is a cationic polymer, which

Correspondence: Dr K Regnström, Department of Pharmacy, Uppsala University, PO Box 580, SE-751 23 Uppsala, Sweden Received 7 January 2003; accepted 10 March 2003 has been shown to be one of the most effective DNA delivery system known, both to cells in culture and in vivo, ${ }^{8}$ shuffling the DNA directly to the nucleus ${ }^{9,10}$ and with high-level gene reporter activity already $6 \mathrm{~h}$ after administration. ${ }^{11}$ PEI has also been shown to be a very effective gene delivery vehicle for lung transfection, producing high antibody titers against the encoded protein. ${ }^{12}$

However, the use of PEI as a gene delivery system in humans is hampered by its well-known toxicity. We have therefore used PEI as positive control in a toxicogenomics analysis of polycationic gene delivery systems (manuscript in preparation). Toxicity is the reason why no clinical studies with PEI are currently performed, with the consequence that not very much is known about the types of immune responses evoked by PEI, besides its ability to activate the complement system upon intravenous administration. ${ }^{13}$ However, because PEI is the one of most effective gene delivery system known and because we wanted to use the information gained in the development of efficient, but safer gene delivery systems, we decided to study PEI's ability to stimulate a systemic immune response when formulated with or without a LacZ - reporter gene plasmid and administered intratracheally to mice.

\section{Results and discussion}

In this study, we used a similar experimental approach as reported earlier, ${ }^{14}$ which is a comparison between the 
gene expression profiles of the adjuvant-antigen complex, here PEI formulated with LacZ (PEI+), with that of the formulation alone (PEI-) and that of non-immunized mice (N). In detail, the reporter plasmid CMVSPORT- $\beta$ gal, which encodes the gene for the LacZ protein, was formulated with PEI (PEI+). The DNA vaccine or the formulation without plasmid was administered intratracheally to mice at two different doses according to a standard immunization protocol, using non-immunized mice as controls for all four groups. After a booster dose, spleen cells were harvested and pooled for each formulation ( $n=3-4$ mice) to diminish interindividual variation effects. ${ }^{14-16}$ The spleen lymphocytes were isolated and restimulated in vitro with LacZ antigen peptide for 6 days and a second time for 4 and $24 \mathrm{~h}$ before RNA purification. These time points have been verified earlier as ideal for the study of cytokine responses on the gene expression level. ${ }^{14,16}$ Spleen lymphocytes of PEI- mice and the non-immunized mice were cultured for the same time periods, but not in vitro restimulated with antigen in order to avoid both potential non-specific and specific effects of the LacZ antigen. Also, we had observed earlier that in vitro restimulation of lymphocytes from non-immunized mice with tetanus toxoid antigen did not result in expression changes of genes other than those that are involved in mitotic effects. ${ }^{14}$ RT-PCR confirmation of the array results was not performed, because earlier we have found a good correlation between the gene expression results of the array used and RT-PCR results. ${ }^{14,16}$

\section{Dose-dependent effects}

Mice given a $67.5 \mu \mathrm{g}$ dose of PEI without plasmid did not survive the administration procedure despite repetition of the experiment. This is not surprising as it is well known that PEI itself is toxic because of its ability to permeabilize membranes. ${ }^{17}$

In order to identify the optimal administration dose for the systemic immune response, PEI+ samples obtained from administration of the lower $(33.75 \mu \mathrm{g})$ and the higher $(67.5 \mu \mathrm{g})$ dose were compared concerning the expression levels of genes identified earlier to be important in the systemic immune response of the tetanus and diphtheria toxoid vaccines. ${ }^{14,16}$ In summary, gene expression levels from the higher dose PEI+ samples were much lower as compared to lower dose PEI+ samples (data not shown). To name the most important genes: the markers for antigen-specific $\mathrm{CD} 8^{+} \mathrm{T}$ cells, SATB1 and lung Kruppel-like factor (LKLF), the costimulatory signal B7-2 (CD86) on antigen-presenting cells, the early T-cell activation signal Syk, the T-cell activation antigens CD2 and LFA- $1 \propto$ (CD11a) and CD4 receptor as well as genes involved in the cytokine regulation for both the Th1 and Th2 responses (data not shown). Therefore, samples derived from mice given the higher doses of DNA vaccine were excluded from further analyses.

\section{Number of activated genes in global gene expression analysis}

Not much is known of the systemic immune response of PEI alone or formulated with a plasmid. In fact, no articles studying their immune response reactions after administration could be found in the literature. We

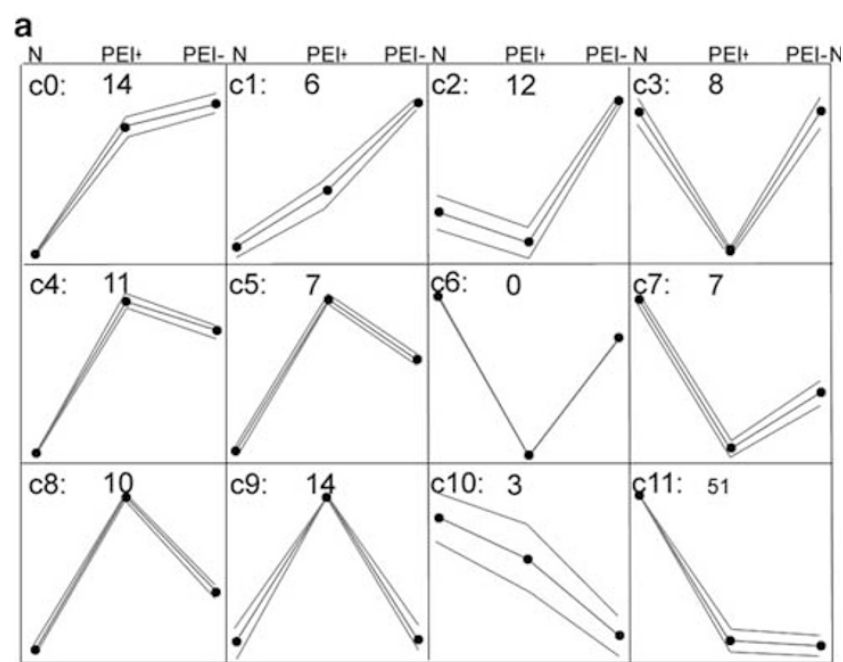

b

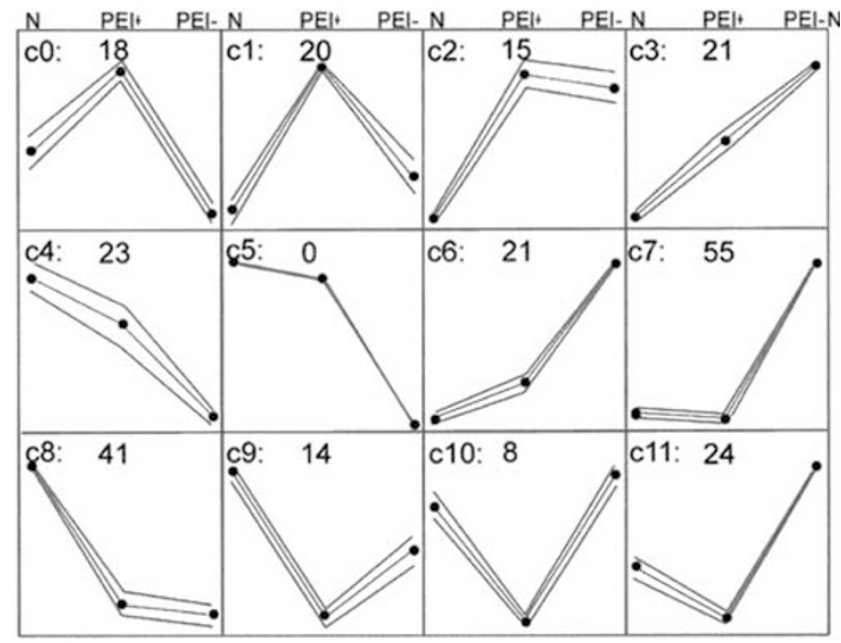

Figure 1 Gene expression differences in spleen cells between mice treated with PEI+, PEI- and nontreated mice (N) after the second in vitro restimulation with LacZ peptide for $4 h(a)$ and $24 h(b)$. The differences are shown as self-organizing map clusters according to Tamayo's algorithm ${ }^{18}$ using the criteria for significant gene expression described in Materials and Methods. (a) A total of 143 genes were found in 12 clusters (c0-c11) containing between 3-51 genes at $4 \mathrm{~h}$ and (b) 260 genes in 12 clusters ( $c 0$ c11) containing between 8 and 55 genes at $24 \mathrm{~h}$. Lines connecting the dots indicate the mean expression profiles; outer lines indicate the s.d. A complete list of the genes in each cluster can be obtained in the supplementary material at www.farmfak.uu.se/farm/pharmacogenomics.html.

therefore compared the gene expression profiles of PEIand PEI+ mice spleen lymphocytes with the profiles obtained from non-immunized mice at each time point after in vitro restimulation by cluster analysis. ${ }^{18} \mathrm{~A}$ total of 12 clusters were obtained that contained genes with significant gene expression changes at each time point (Figure 1).

Counting the total number of genes with significant gene expression changes of all the clusters at each time point after in vitro restimulation with antigen, it was found that the total number was almost two times higher in the $24 \mathrm{~h}$ sample as compared to the total number of the 4 h samples (Table 1 ).

Two explanations are plausible: (1) the systemic immune response after immunization with PEI+ follows different kinetics as compared to the clinically used 
Table 1 Number of genes with significant gene expression changes as assessed from Figure 2

\begin{tabular}{lllccc}
\hline & Total (\%) & N (up) & PEI+ (up) & PEI+ (down) & PEI- (up) \\
\hline $4 \mathrm{~h}$ & $143(34)$ & $61(10)$ & $52(9)$ & $7(1)$ & $32(5)$ \\
$24 \mathrm{~h}$ & $260(44)$ & $55(9)$ & $53(9)$ & $22(4)$ & $121(21)$ \\
\hline
\end{tabular}

The percentage of expressed genes (\%) is calculated from the total number of genes on the array $(n=588)$, The sum of the number of genes with significant gene expression changes from all samples does not necessarily correspond to the total number of genes, since genes can be activated in several samples at the same time.

tetanus- and diphtheria-toxoid vaccines because of the different antigens used; (2) different kinetics between the antigen-adjuvant combination and the adjuvant alone as compared to the clinically used vaccines. Therefore, the number of genes, which were up- or downregulated in each category, was counted for each time point (Table 1). This showed that the main contributor to the difference in the total number of genes between the two time points is PEI-, while the gene numbers for PEI+ vaccine did not change significantly. This is in contrast to our earlier observations derived from the analyses of the systemic immune response in spleen lymphocytes from mice after immunization with tetanus toxoid or diphtheria toxoid vaccines, ${ }^{14,16}$ where an early burst of gene expression during the immune response has been shown. However, only a smaller part of these genes were found to be involved in immune response reactions at $24 \mathrm{~h}$ in PEI-, while the major part of these genes were found to be involved in cellular processes other than immune response.

In conclusion, there is a difference in the kinetics of gene activation in the PEI+ vaccine in contrast to the early burst of gene expression in tetanus- and diphtheriatoxoid vaccines. ${ }^{14,16}$ This might be a characteristic of DNA vaccines in general or account for this specific antigen-adjuvant combination.

\section{Immune response}

Genes activated in PEI+ mice. Our earlier studies ${ }^{14,16}$ have shown that mRNA expression analysis can be used to differentiate between Th1 and Th2 response, as well as adaptive and innate immune response. Therefore, special interest was put into the analysis of gene activation involved in immune response in PEI+ mice. The expression levels of marker genes, which have been identified in the earlier studies, are shown in Figure 2a and b. See also Figure 1a, clusters c4, c5, c8 and c9; Figure $1 b$, clusters $c 0$ and $c 1$. All of these genes were expressed at several times at lower levels in non-immunized mice, as expected (data not shown).

Earlier identified cytokine markers for a Th1 response $^{14}$ were upregulated (Figure 2a): Inf $\gamma \mathrm{R}, \mathrm{YB} 1$ and Jak3, markers for interferon $\gamma$, as well as SLAP, which is necessary for TCR-driven IL-2 transcription. The activation of JunD and Stat 3 indicates an elevated level of IL-5. The levels of these markers were about two to three times higher than the levels reported earlier for the clinically used, intra-muscularly administered tetanus toxoid vaccine with aluminum phosphate as adjuvant. ${ }^{14}$ Therefore, a strong mixed Th1-Th2 response is apparent. a

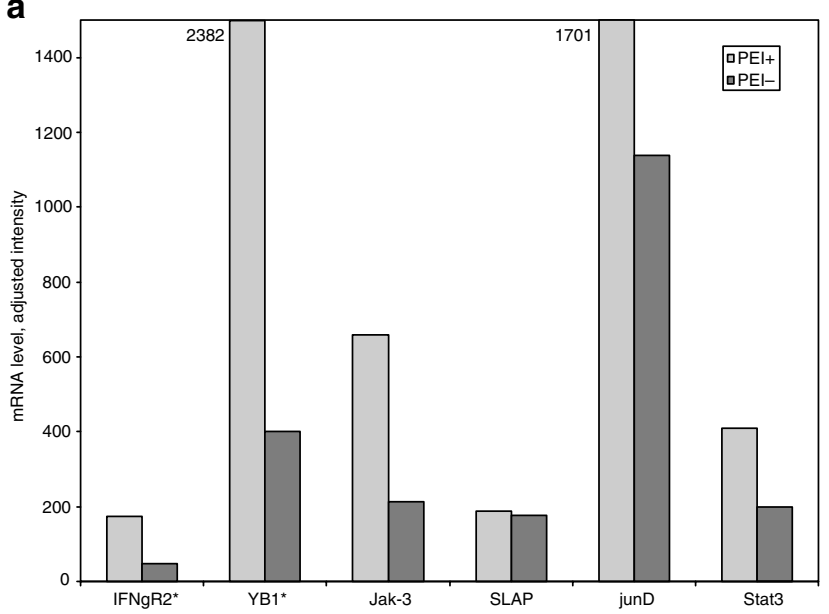

b

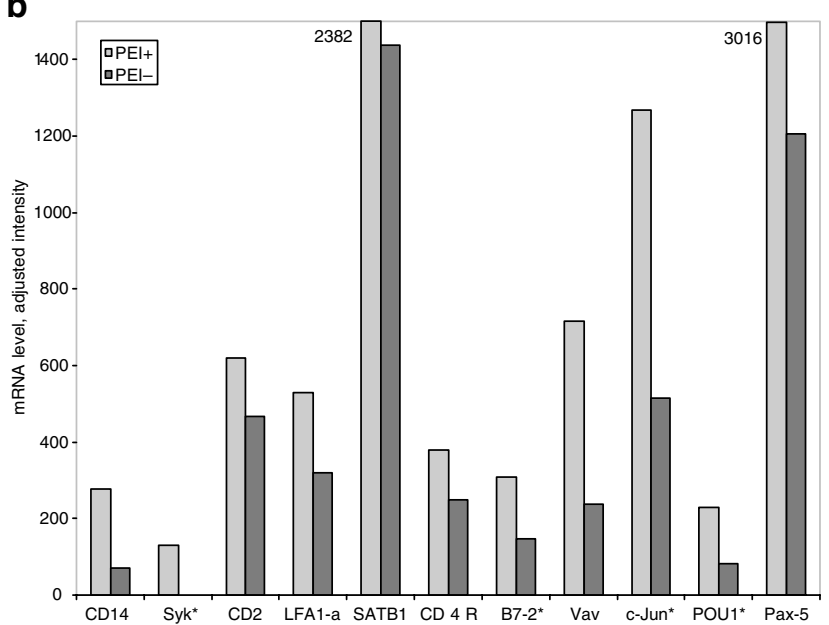

C

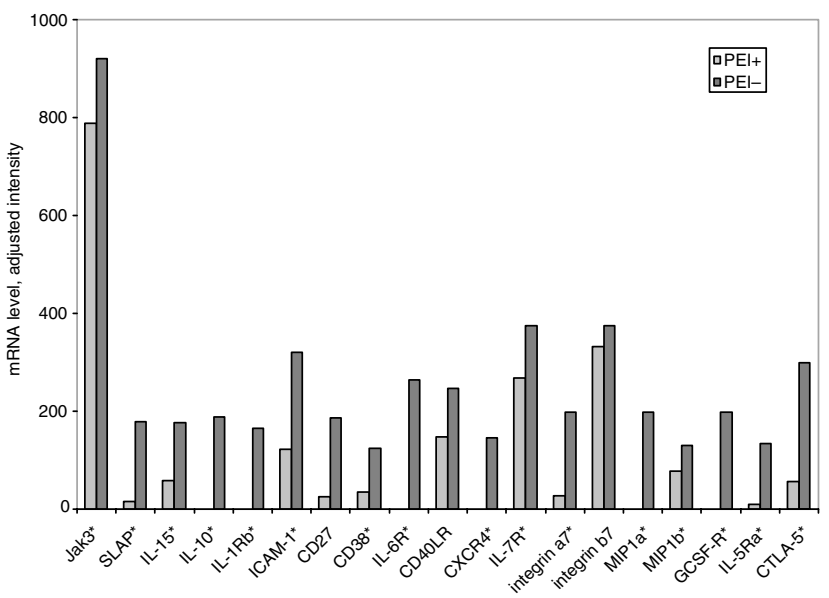

Figure 2 Expression of genes involved in immune response. The mRNA levels are shown as adjusted intensities for each gene as defined in the Materials and methods section. (a) Upregulated markers for the Th1 and Th2 response and (b) of the adaptive and innate immune response in spleen cells from PEI+-immunized as well as PEI--treated mice. (c) Upregulated genes involved in immunogenicity and immunostimulation in PEI-treated mice as compared to PEI+-immunized mice. Only the highest gene expression levels were shown if the gene was found both at 4 and $24 \mathrm{~h}$ after in vitro restimulation, with genes expressed at higher levels at $24 \mathrm{~h}$ marked by * 
Upregulated genes involved in the adaptive immune response in the presence of antigen were the monocytic LPS receptor CD14, the early T-cell activation signal Syk, the T-cell activation antigen CD2, LFA-1 $\alpha$, the marker for $\mathrm{CD}^{+} \mathrm{T}$ cells, SATB1, and CD4 ${ }^{+} \mathrm{R}$ (Figure 2b). Furthermore, the costimulatory signals B7-2 (CD86) on antigenpresenting cells and c-Jun, the POU domain associated factor 1, which is involved in T-cell antigen-dependent Bcell maturation, and Pax-5, important for committed Bcell differentiation, were upregulated.

In summary, genes indicative of a mixed Th1/Th2 response with activation of both CD8 and CD4 T cells including the important costimulatory signal B7-2 as well as B-cell differentiation were observed. Therefore, it can be concluded that the intratracheal administration of PEI+ vaccine according to a traditional immunization scheme resulted in a systemic immune response as observed in the gene expression profiles of mice spleen lymphocytes.

PEl is immuno-stimulatory in the absence of formulated plasmid DNA. As seen in Figure $2 \mathrm{a}$ and $\mathrm{b}$, the levels of the genes involved in the Th1 and Th2 response and the adaptive immune response were activated at considerably high levels in PEI-, but these levels were still lower as compared to PEI+. This indicated an ability of PEI to activate certain immune response processes even in the absence of DNA. Therefore, the data were searched for any further evidence of PEI activation of the immune response.

The clusters in Figure 1a and $b$ were analyzed for those showing higher upregulation in PEI- as compared to all other samples. The clusters $\mathrm{c} 0, \mathrm{c} 1$ and $\mathrm{c} 2$ in Figure $1 \mathrm{a}$ and c3, c6, c7 and c11 in Figure 1b were upregulated in PEI-. With one exemption (CD40L R; see below) only the clusters of the $24 \mathrm{~h}$ sample contained immune response marker genes at higher levels as PEI+. These genes are shown in Figure 2c.

They comprise earlier identified T-helper cell response markers ${ }^{14}$ as well as other immune response genes: Jak-3, a marker for interferon gamma, SLAP, which is found in TCR-driven IL-2 transcription, the IL-2-like cytokine IL15 , which activates proliferation and cytotoxicity of $\mathrm{CD} 8^{+}$ memory cells in an antigen-independent way, ${ }^{19}$ the Th2 response gene IL-10 and the IL-1 receptor $\beta$. Furthermore, the following costimulatory signals were found: ICAM-1 (CD54) on antigen-presenting cells, CD22 on B cells, CD27 on B and T cells and CD38, which is found on activated $\mathrm{T}$ cells and known to stimulate B-cell proliferation. Upregulated genes, which are known to be reminiscent of B-cell activation, were IL-6R and CD40L $R$. Other genes, which are involved in immune response reactions, were activated in PEI-: CXCR-4, a chemokine receptor on $\mathrm{CD}^{+}$T cells; IL-7R, regulating the pre-B- and -T-cell growth; the adhesion molecules integrin $\alpha 7$ and $\beta 7$, known to direct armed effector $\mathrm{T}$ cells to the site of infection; MIP1 $\alpha$ and MIP1 $\beta$, which are chemokines promoting Th1-dominant environments; GKLF; GCSF-R, which is involved in neutrophil development and differentiation; the macrophage mannose receptor involved in the identification of pathogens; Il-5R $\alpha$ on $T$ cells, necessary for eosinophil differentiation; and several granzymes, involved in CTL responses in mice.

Many of these genes were hitherto only found activated in the presence of antigen ${ }^{14,16}$ and not in systemic responses toward adjuvant only (aluminum phosphate). ${ }^{14}$ However, the absence of gene expression marking costimulatory signals for T cells together with Tcell activation suggests that a systemic immune response to PEI- is not activated. Instead it is proposed that PEI, in the absence of antigen-encoding DNA, has important immuno-stimulatory abilities, which has not been previously reported in the literature.

In order to understand the relation between all immune reponse genes upregulated in the PEI- sample, the genes were subjected to the bioinformatic tool PubGene. ${ }^{20}$ This software extracts gene-to-gene cocitations from over 10 million Medline records and presents their relation as a network. Since this tool is only available for human genes, we translated the mouse gene symbols to human gene symbols using the database GeneCard of the Weizman Institute (http://bioinformatics.weizmann.ac.il/cards). ${ }^{21}$ A network was obtained, which contained all genes besides ITGB7 (data shown at www.farmfak.uu.se/farm/pharmacogenomics.html). Submitting the genes of the network to PubMeds MeSh Map tool (www.nlm.nih.gov/mesh), the three highest ranked returned term associations for these genes were somatomedins-blood (insulin-like growth factor 1 (IGF1)) (score of 0.75$)$, chemokines (0.73) and granulocyte colony stimulating factor (GCSF) genetics (0.70). The annotation to IGF-1 suggests also involvement in mitogenesis and antiapoptotic effects. ${ }^{22}$ IGF-1 has lately also been linked to the development of colorectal cancer in humans and mice. ${ }^{22}$

This supports our hypothesis that PEI- has important immuno-stimulatory abilities, stimulating both Th1 and Th2 responses via GSCF. Further support can also be found in a comparison of the gene expression levels of markers for CD4 and CD8 in PEI-, + and PEI+. PEIand + have comparable expression levels in both markers. Formulation and DNA together (PEI+) show expression of these markers at almost double levels at $4 \mathrm{~h}$ after in vitro restimulation with antigen.

\section{PEl+ is superior to traditional vaccines in immune} response gene activation. As stated above, some of the immune response genes were found at higher gene expression levels as compared to the clinically used tetanus toxoid vaccine. However, the literature is full of articles saying that DNA vaccines are less immunogenic than protein vaccines. ${ }^{22}$ We therefore compared the gene expression profiles of PEI+ with those of mice immunized with tetanus toxoid formulated on aluminum phosphate $\left(\mathrm{TT}_{\mathrm{ADJ}}\right)^{14}$ and non-immunized mice at each time point after in vitro restimulation with respective antigen. We speculated that a comparison was possible since systemic immune responses were analyzed, both types of vaccines were administered according to the same administration scheme have at different sites, and an in vitro restimulation with respective antigen was performed. Using the GeneCluster program ${ }^{23}$ the comparison resulted in 12 clusters for each time point with about $25 \%$ of all genes on the array showing significant gene expression differences (Figure 3). No obvious differences in the gene expression levels and kind of genes, which are involved in immune response, were obtained comparing the profiles at $24 \mathrm{~h}$ after restimulation (data not shown). Therefore, only the data 
a

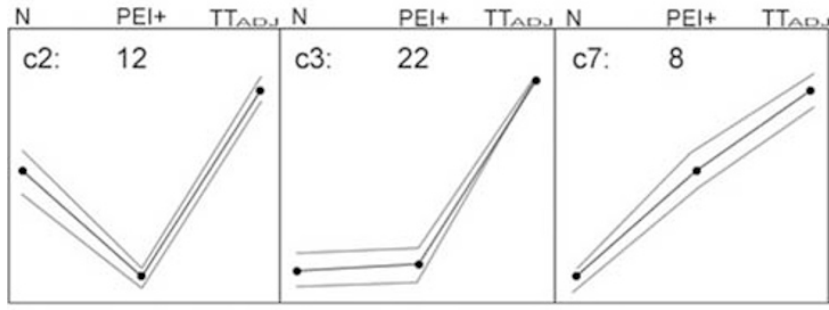

b

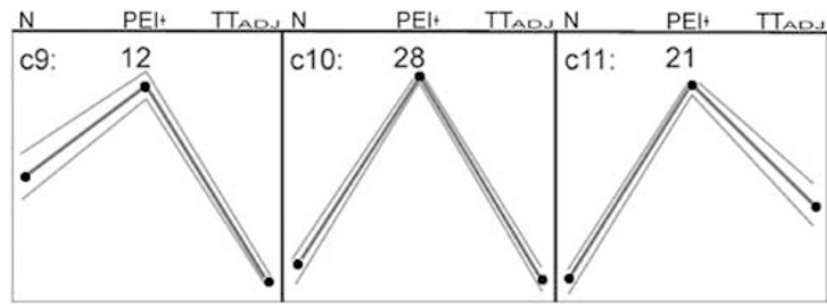

Figure 3 Gene expression differences in spleen cells between PEI+-,

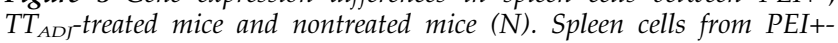
treated mice were in vitro restimulated by LacZ peptides for 6 days and a second time for $4 \mathrm{~h}, \mathrm{TT}_{\mathrm{AD} \text { - }}$-treated mice were in vitro restimulated by tetanus toxoid for $4 \mathrm{~h}$. Self-organizing map clusters according to Tamayo's algorithm ${ }^{18}$ using the criteria for significant gene expression described in Materials and methods are shown. 167 genes were found in 12 clusters (c0-c11) containing 6-28 genes. Only clusters containing genes highly activated in (a) $T T_{A D J}$ and (b) PEI+ are shown. Lines connecting the dots indicate the mean expression profiles; outer lines indicate the s.d. A complete list of the genes in each cluster can be obtained in the supplementary material available at our website at www.farmaci.uu.se/..

of the gene expression profile of the $4 \mathrm{~h}$ samples are shown. Three clusters were found to contain 61 genes with higher gene expression levels in PEI+ (c9, c10 and c11) as compared to $\mathrm{TT}_{\mathrm{ADJ}}$, which was found with higher gene expression levels in clusters c2, c3 and c7 with a total of 42 genes.

Comparing the type of immune response genes represented in either group of genes, the PEI+ samples showed superiority in higher gene expression levels of immune response markers as well as the activation of a greater number of genes (22 genes) as compared to the $\mathrm{TT}_{\mathrm{ADJ}}$ sample (10 genes). To name the most important ones, PEI+ had the highest levels of the interferon gamma markers YB1 and Jak-3, ${ }^{14}$ the IL-5 marker Stat3, and markers of the adaptive immune response such as the monocytic LPS-receptor CD14, the T-cell activation antigen CD2, LFA- $\alpha$, the CD4 receptor, the costimulatory signal B7-2 (CD86) on antigen-presenting cells and the Bcell differentiation marker Pax-5. TA instead had higher levels of gene expression in the costimulatory signal for $\mathrm{B}$ cells, CD22, and the marker for antigen-specific CD8 ${ }^{+} \mathrm{T}$ cells, SATB1. The lower activation of B-cell costimulatory signals in $\mathrm{PEI}+$ as compared to $\mathrm{TT}_{\mathrm{ADJ}}$ is in agreement with the earlier observation that the major effector cells in mucosal immune responses consist of $80 \%$ in $\mathrm{T}$ lymphocytes and only $20 \%$ IgA B cells. ${ }^{24}$ The most remarkable difference between these two gene expression profiles can be seen in the different types of T-cell activation mechanisms. While the higher upregulation of CD4R in PEI+ suggests that the LacZ antigens were to a large extent processed via the MHC II pathway, the higher expression level of SATB1 in $\mathrm{TT}_{\mathrm{ADJ}}$ points out that the $\mathrm{TT}_{\mathrm{ADJ}}$ antigens were processed and presented more via the MHC I pathway. This is in contrast to what has been proposed for DNA vaccines, which is predominantly a CD8+ response, ${ }^{25,26}$ despite the fact that PEI+ shows higher interferon gamma marker levels as compared to $\mathrm{TT}_{\mathrm{ADJ}}$. However, the plasmid used in our immunizations does not contain CpG motifs, which are commonly considered to be responsible for the Th1 biasing effect. ${ }^{27}$ Also, a comparison of the gene expression profiles in lymphocytes of mice immunized intratracheally with PEI+ or the plasmid only did not show any higher gene expression levels of SATB1 in the + sample (data not shown). Studies of the immune response after intra-nasal administration with cholera toxin B subunit in humans also showed that a CD4 ${ }^{+} \mathrm{Th} 2$ response was induced, pointing toward differences in immune responses correlating to the administration route. ${ }^{28}$ Taken together, the observed lower $\mathrm{CD}^{+}{ }_{-}$ activating effects of PEI in the presence of DNA seem reasonable.

\section{Activation-induced cell death}

During memory development and effector expansion of rare antigen-specific T-cells within 0.5-2 days after antigen stimulation apoptotic processes play an important role in eliminating $\mathrm{T}$ cells, which are not specific for the inducing antigen-a process known as activationinduced cell death (AICD). ${ }^{29}$ Several regulators are discussed in the literature: ${ }^{30}$ Fas, which regulates the number of lymphocytes with TCR $\gamma \delta$, and the apoptosis inhibitor Bcl-2, which mediates the survival of primed $\mathrm{T}$ cells. We had observed earlier an involvement of $\mathrm{Bcl}-2$ in the gene expression profiles of the clinically used tetanus vaccine. ${ }^{14}$ Analyzing the gene expression data of PEI+ at $4 \mathrm{~h}$ and $24 \mathrm{~h}$ after the in vitro restimulation with antigen, we found that Bcl-2 was not upregulated. Furthermore, two antagonists of Bcl-2 and members of the Bcl-2 family, $\mathrm{BAK} 1^{31}$ and Bcl-xs, ${ }^{32}$ were upregulated. Because of these data and reports from the literature that apoptotic processes are regulated by competitive dimerizations between different agonists and antagonists, ${ }^{33}$ it is proposed that an upregulation of $\mathrm{Bcl}-2$ is not responsible for the survival of antigen-specific $\mathrm{T}$ cells in spleen lymphocytes, when mice were immunized with PEI+.

Instead, we found that PEI+ activated the expression of FasL, an apoptosis promoter, which is involved in cytotoxic T-cell-mediated apoptosis and in the antigenstimulated suicide of mature T cells. ${ }^{30-31}$ FasL together with TCR is also known to induce the expression of TRAIL, ${ }^{36}$ which was also found upregulated in PEI+. To summarize, the data show that it is likely that FasL mediates the activation-induced cell death in spleen lymphocytes, isolated from mice immunized with PEI+ after intra-tracheal administration.

In conclusion, we propose that different vaccines, or different antigen-adjuvant combinations, activate a different type of regulator during activation-induced cell death. Currently, three types are known: We found Bcl-2 as an inhibitor of cell death in primed T cells activated in the clinically used tetanus toxoid aluminum phosphate vaccine. ${ }^{14}$ Mitchell et $a l^{37}$ showed that the survival factor Bcl-3 is upregulated in lymphocytes from mice that had been given either LPS or vaccinia virus. Here in this study, the FasL-mediated death pathway for removal of 
naive $T$ cells is activated in lymphocytes of mice that were immunized with PEI+.

We further speculate that the factor governing the type of regulator for activation-induced cell death (AICD) is the way the antigen is presented: Pronounced $\mathrm{CD}^{+}-$ mediated immune responses with inflammatory characteristics activate the Bcl-2 survival pathway as we reported earlier for the tetanus toxoid vaccine. ${ }^{14}$ Immune responses mediated via predominantly $\mathrm{CD}^{+}$instead control AICD via FasL as observed in this study (PEI+) and as reported earlier. ${ }^{38}$ Finally, vaccines consisting of other types of adjuvants, such as lipopolysaccharides, induce the Bcl-3 survival pathway. ${ }^{37}$ Since it was reported by Rebello $e a^{39}$ that IL-4 deprivation leads to induction of Bcl-3 expression, it can be assumed that all IL-4 affecting adjuvant and antigen-adjuvant combinations induce Bcl-3-mediated survival of primed $\mathrm{T}$ cells. The data presented here also suggest that the mechanisms governing immune responses are much more complex and can differentiate between different antigens and adjuvants in a much more sensitive way than recognized earlier.

\section{Genes involved in other cellular processes}

As stated above, 121 genes were activated in PEI- at $24 \mathrm{~h}$ after in vitro restimulation with antigen peptides (Table 1). Only a small fraction of these genes (a total of 23 genes or 18\%) could be linked to immunological responses. In an earlier study, which compared the gene expression profiles of mice spleen lymphocytes after administration of the clinically used tetanus toxoid formulated with aluminum phosphate as adjuvant or the adjuvant alone, we had observed that adjuvant without antigen present could activate 47 genes associated with inflammation, apoptosis, stress and oncogenesis and it was proposed that these genes could be part of danger signals of the innate immune response. ${ }^{14}$ Therefore, we wanted to analyze if the large number of genes, which were activated in PEI- and suppressed in PEI+, also could be associated with the danger model.

After clustering the gene expression data (Figure 1) genes activated in PEI- were found in the clusters $\mathrm{c} 1$ and c2 of the $4 \mathrm{~h}$ sample (Figure $4 \mathrm{a}$ ) and in the clusters c3, c6, $\mathrm{c} 7$ and $\mathrm{c} 11$ for the $24 \mathrm{~h}$ sample (Figure $4 \mathrm{~b}$ ). At a first glance, the over-representation of receptor expression (a number of 24) in c3 but also c6 and mainly c7 of the $24 \mathrm{~h}$ sample was obvious. However, the receptors seem to be involved in different cellular processes. In order to understand the combined functions of the receptors and the other genes activated by PEI-, we used the literature association tool PubGene ${ }^{20}$ (see above). Since it has been proposed earlier that clusters are reminiscent of co-regulated genes involved in the same processes ${ }^{40}$ and the entry amount of data is limited, we subjected the gene list of each cluster to the PubGene tool.

The obtained networks from each cluster were examined for participation in the literature network and nonparticipating genes were excluded (66 genes out of 139 genes). The literature subnetworks for each cluster may be searched at our website at www.farmfak.uu.se/ farm/pharmacogenomics.html. The remaining 73 genes were submitted to the MeSh Map tool. The four returned term associations with the highest scores for these genes were serotonin genetics (1.00), protein kinase C genetics (1.00), glutathione transferase genetics (1.00) and Alzhei- mer Disease genetics (0.91). The relations between serotonin and protein kinase $C$ to the neurodegenerative Alzheimer Disease are well known in the literature, while an involvement of glutathione transferase has not been established.

It has been speculated that free PEIs exhibit toxic effects on cells because of their ability to permeabilize plasma membranes. ${ }^{41,42}$ Alzheimer's disease is known to be associated with the formation of fibrils from mutant amyloid proteins. In a recent publication it was found that these fibrils are very similar to pore-forming toxins and the authors suggest that inappropriate membrane permeabilization is the cause of cell death and dysfunction in Alzheimer's disease. ${ }^{43}$ Godbey et a ${ }^{44}$ state that free PEI leads to growth inhibition and cell death and that removal of free PEI by formulation with DNA decreases toxicity.

Taken together, PEI- activates genes involved in cellular processes such as cell cycle regulation, oncogenesis and differentiation, indicating toxic characteristics of free PEI and the danger of adverse reactions after immunization with PEI-containing vaccines. This is supported by the fact that mice in our study given the higher dose of PEI without DNA plasmid present died shortly after the booster administrations.

Comparing the gene expression profiles in mouse spleen lymphocytes caused by PEI- administration to lung mucosa and the one caused by aluminum phosphate administration to muscle, we were not able to detect many similarities, beside four genes, which can be associated with immune response reactions: TFIID, identified as possibly involved in immune responses in one of our earlier studies; ${ }^{16}$ SLAP, which is necessary for TCR-driven Il-2 transcription; the GCSF receptor, necessary for the production of granulocytes, macrophages and dendritic cells; and cathepsin B, involved in antigen processing in endocytic vesicles. Obviously, each adjuvant elicits different systemic responses involved in immune response or other cellular processes, but this could be partly caused by the different administration routes, i.m. for aluminum phosphate and i.t. for PEI. This is in line with our earlier observation that each adjuvantantigen combination activates a different gene expression profile in a systemic response. ${ }^{16}$

However, one similarity to the tetanus vaccine could be found. This is the down-regulating effect of antigenin the case of PEI-LacZ formulation encoded in the plasmid-on the expression levels of those genes, which were highly upregulated if only the adjuvant is administered (Figure 4, Figure 1a clusters $\mathrm{c} 1$ and $\mathrm{c} 2$, Figure $1 \mathrm{~b}$ clusters c 3 and c7). Since the protein encoded in the DNA plasmid is not present at the time of administration in contrast to the tetanus vaccine, it is concluded that suppressive effects of the antigen are not exerted at the time of administration but during the presentation of the antigen in immune response reactions.

This supports our earlier proposal that adjuvant elicits responses which are part of the endogenous danger signals in the innate immune system and that this modulation by the antigen is necessary to protect against adjuvant-mediated effects. ${ }^{14}$ This greatly supports the danger theory postulated by Matzinger et al. ${ }^{23}$ It also appears to be a general mechanism applying to all types of antigens in vaccines, protein and DNA, studied by us so far. $^{14,16}$ 
Obviously, this adjuvant-modulating capacity of antigens is of great importance for the safety of vaccines and protection against possible unwanted adverse reactions caused by the adjuvant. However, it is not clear if the adjuvant-modulating capacity of antigens is not only coupled to the presentation of the antigen but also to the strength of the antigen's immunogenicity. If this is the case, the often poorly immunogenic antigens produced by recombinant techniques present an obvious danger for adverse reactions. In the case of DNA vaccines, the danger lies in the time interval between the delivery of the DNA and the synthesis/presentation of the antigenic protein. Therefore, gene delivery systems with rapid onset of gene expression and translation are preferable as is the case with PEI. Further research of this interplay is needed to develop new, safe and more effective vaccine adjuvants. a

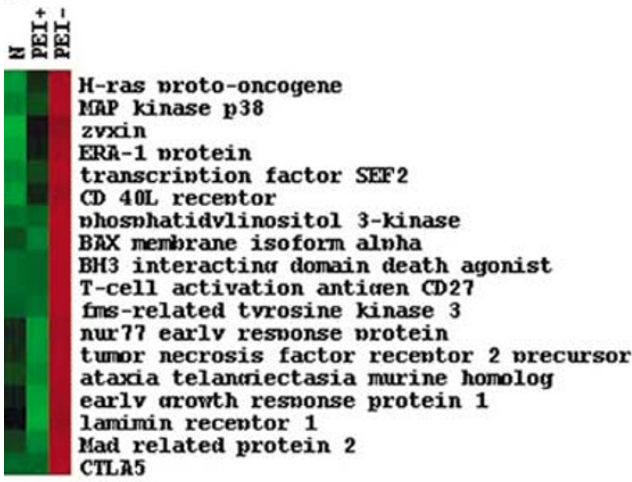

b

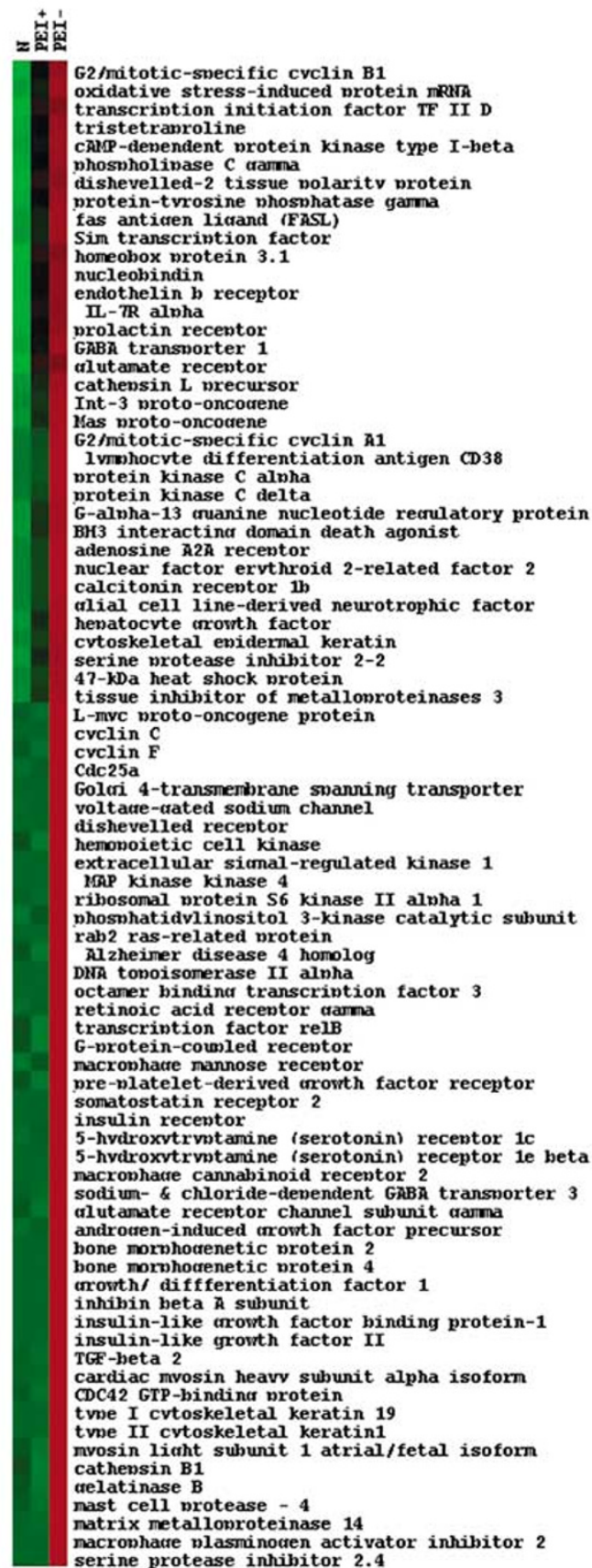

Figure 4 Clustergram of the gene expression profiles from the six clusters $c 1$ and $c 2$ in Figure $1 a(a)$ and $c 3, c 6, c 7$ and c11 in Figure $1 b$ (b) obtained using the Treeview program. ${ }^{11}$ The sample is indicated at the top of each column. Red indicates upregulated and green downregulated genes, respectively. (c) Functional categories of genes significantly activated in PEI-. The information obtained by clustering (Figure 1) was used to assess the involvement of the genes in different cellular processes as specified by the array manufacturer. The percentage of each category compared to the total number of activated genes is shown. The cells were cultured for $4 h(A)$ and $24 h(B)$. 
C

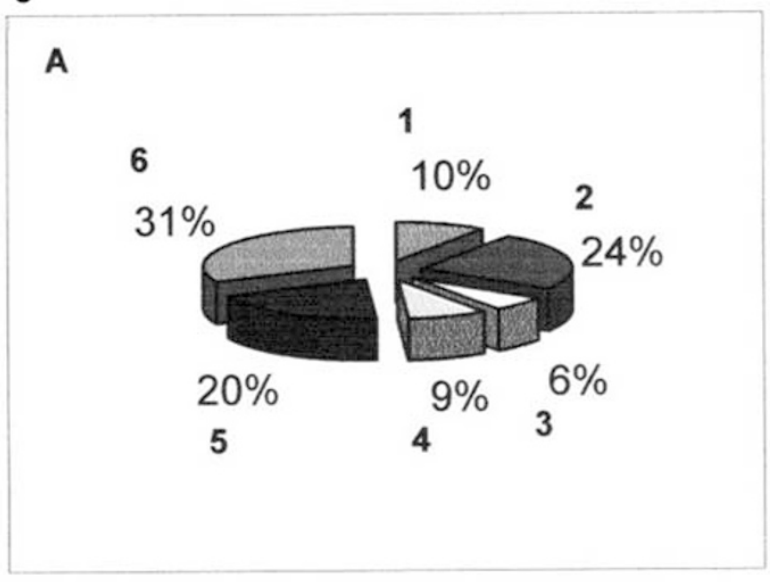

B

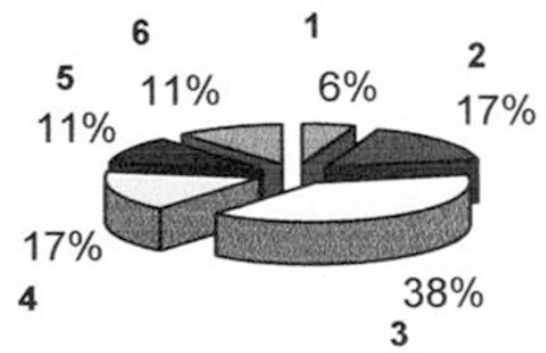

1 Oncogenes, Tumor suppression, Cell Cycle

2 Stress response, ion channel, signal transduction

3 Apopotosis, DNA metabolism

4 Transcription factors, DNA binding proteins

5 Cytokines, Growth factors, adhesion

6 Cell-cell communication, cytoskeleton

Figure 4 Continued

\section{Materials and methods}

\section{Animal experiments}

The animal experiments were approved by The Swedish National Board for Laboratory Animals (the local ethical committee). Male Balb/c mice, 8-10 weeks old (Charles River), were randomly separated into groups of three to four animals. The mice were given an intra-tracheal dose of DNA vaccine containing 50 or $100 \mu \mathrm{g}$ of endotoxin-free LacZ plasmid (pCMVSport. $\beta$-gal, Invitrogen) (obtained from Aldrevon) formulated with 33.75 or $67.5 \mu \mathrm{g}$ PEI polymer (PEI+) $25 \mathrm{kDa}$ (Sigma) at a charge ratio of 5:1-/-. Other groups of mice were given the same doses of PEI without plasmid (PEI-). The same doses were administered 2 weeks later. At 3 weeks after the booster, spleens were isolated, pooled for each group to compensate for individual genetic variation, and washed in cold PBS.

\section{Cell culture experiments}

A single-cell suspension from the spleens was prepared and erythrocytes were removed by incubation in $1 \mathrm{M}$ Tris buffer, pH 7.2 on ice for $10 \mathrm{~min}$. The cells were washed twice with RPMI medium (Dulbecco) supplemented with $292 \mu \mathrm{g} / \mathrm{ml}$ L-Gln, $50 \mu \mathrm{M}$ 2-mercaptoethanol, $100 \mathrm{IU} / \mathrm{ml}$ penicillin, $100 \mu \mathrm{g} / \mathrm{ml}$ streptomycin and $10 \%$ heat-inactivated FCS (Gibco). Aliquots of the cells $\left(1.25 \times 10^{6}\right.$ cells $\left./ \mathrm{ml}\right)$ were plated into cell culture flasks and incubated for 6 days, at $37^{\circ} \mathrm{C}$ in $5 \% \mathrm{CO}_{2}$ at approximately $90 \%$ humidity with the antigen peptide for LacZ beta-galactosidase peptide ${ }_{876-884}$ (TPRPARIGL; 99\% purity) (obtained from the Core Facility for Protein/ DNA Chemistry, Queens University, Kingston, Canada) at a dose of $1 \mu \mathrm{g} / \mathrm{ml}$ cell culture medium, followed by a second incubation with the same peptide for 4 or $24 \mathrm{~h}$, respectively. The control groups of mice, which had received PEI alone, were cultured but not in vitro restimulated with peptide antigen. The in vitro restimulation with pure tetanus toxoid (GMP grade, SBL vaccine, Solna, Sweden) was performed as reported earlier. ${ }^{14,16}$ After restimulation, the cells were washed with cold PBS and used for RNA isolation.

\section{Analysis of gene expression by array technology}

Total RNA was purified from splenocyte cells using the Ambion's Totally RNA ${ }^{\mathrm{TM}}$ kit. DNA contamination was removed using DNAse I (Promega). Atlas mouse cDNA expression arrays with 588 genes (Clontech) were used according to the manufacturer's guidelines and the reproducibility was assessed as reported earlier. ${ }^{14}$ The level of expression was quantified by phosphorimaging (Packard) and the obtained image data were processed using the AtlasImage program version 1.01a (Clontech). Signals lower than the average background signal were filtered out. The adjusted intensity of each gene was obtained by subtracting the average background signal from the signal intensity. To compare two or more arrays, the adjusted signal intensities of all genes on the arrays were normalized using the global mode and the sum method. Briefly, the ratio of the adjusted signal intensity of gene $Z$ on array 1 to the adjusted intensity of the same gene $Z$ on array 2 was calculated for all the genes on the array and averaged. In order to filter for genes with significant gene expression changes, we used the following criteria: only genes with a difference in signal strength of more than 100 and a more than two-fold expression difference as compared to nontreated control mice in at least one sample were included. ${ }^{14-16}$ Genes were sorted by the program Excel, clustered by the program Genecluster ${ }^{18}$ and visualized by Treeview. ${ }^{45}$ Other bioinformatics analyzers used included GeneCard (http://bioinformatics.weizmann. ac.il/cards), ${ }^{21}$ PubGene $^{20}$ and MeshMap (www.nlm. nih.gov/mesh).

\section{Acknowledgements}

We thank Dr. Diane Burgess, Department of Pharmacy, University of Connecticut for helpful comments. This work was supported by the Swedish Board for Technical Development Grant p11381-1, the Swedish National Network for Drug Development Grant B 6 3368/98 and the Swedish Research Council Grant 6212001-3563. 


\section{References}

1 Brown MD, Schätzlein AG, Uchegbu IF. Gene delivery with synthetic (non viral) carriers. Int J Pharm 2001; 229: 1-21.

2 Ulmer JB et al. Induction of immunity by DNA vaccination: application to influenza and tuberculosis. Behring Inst Mitt 1997; 98: 79-86.

3 Raz E et al. Intradermal gene immunization: the possible role of DNA uptake in the induction of cellular immunity to viruses. Proc Natl Acad Sci USA 1994; 91: 9519-9523.

4 Klavinskis LS, Barnfield C, Gao L, Parker S. Intranasal immunization with plasmid DNA-lipid complexes elicits mucosal immunity in the female genital and rectal tracts. J Immunol 1999;162: 254-262.

5 Gregoriadis G, Saffie R, De Souza JB. Liposome-mediated DNA vaccination. FEBS Lett 1997; 402: 107-110.

6 Singh M, Briones M, Ott G, O'Hagan D. Cationic microparticles a potent delivery system for DNA vaccines. Proc Natl Acad Sci USA 2000; 97: 811-816.

7 Roy K, Mao HQ, Huang SK, Leong KW. Oral gene delivery with chitosan-DNA nanoparticles generates immunologic protection in a murine model of peanut allergy. Nat Med 1999; 5: 387-391.

8 Boussif $\mathrm{O}$ et al. A versatile vector for gene and oligonucleotide transfer into cells in culture and in vivo: polyethyleneimine. Proc Natl Acad Sci USA 1995; 92: 7297-7301.

9 Godbey WT, Wu KK, Mikos AG. Tracking the intracellular path of poly (ethyleneimine)/DNA complexes for gene therapy. Proc Natl Acad Sci USA 1999; 96: 5177-5181.

10 Pollard $\mathrm{H}$ et al. Polyethylenimine but not cationic lipids promotes transgene delivery to the nucleus in mammalian cells. J Biol Chem 1998; 273: 7505-7511.

11 Kichler A, Leborgne C, Coeytaux E, Danos O. Polyethyleneimine-mediated gene delivery: a mechanistic study. J Gene Med 2001; 3: 135-144.

12 Densmore CL et al. Aerosol delivery of robust polyethyleneimine-DNA complexes for gene therapy and genetic immunization. Mol Ther 2000; 1: 180-188.

13 Plank C, Mechtler K, Szoka Jr FC, Wagner E. Activation of the Complement System by Synthetic DNA Complexes: A Potential barrier of Intravenous Gene Delivery. Hum Gene Ther 7: 1437-1446.

14 Regnström $\mathrm{K}$ et al. Tetanus antigen modulates the gene expression profile of aluminum phosphate in spleen lymphocytes in vivo. Pharmacogenomics J 2002; 2: 57-64.

15 Teague TK et al. Activation changes the spectrum but not the diversity of genes expressed by T cells. Proc Natl Acad Sci USA 1999; 96: 12691-12696.

16 Regnström K, Ragnarsson EGE, Artursson P. Expression profiles of clinical relevant diphtheria and tetanus vaccines. Vaccine 2003 (in press).

17 Ferrari S et al. Polyethyleneimine shows properties of interest for cystic fibrosis gene therapy. Biochim Biophys Acta 1999; 1447: 219-225.

18 Tamayo $\mathrm{P}$ et al. Interpreting patterns of gene expression with self-organizing maps: methods and application to hematopoietic differentiation. Proc Natl Acad Sci USA 1999; 96: 2907-2912.

$19 \mathrm{Liu} \mathrm{K}$ et al. IL-15 mimics $\mathrm{T}$ cell receptor crosslinking in the induction of cellular proliferation, gene expression, and cytotoxicity in CD8 ${ }^{+}$memory T cells. Proc Natl Acad Sci USA 2002; 99: 6192-6197.

20 Jensen TK, Laegreid A, Komorowski J, Hovig E. A literature network of human genes for high-throughput analysis of gene expression. Nat Genet 2001; 28: 21-28.

21 Rebhan M, Chalifa-Caspi V, Prilusky J, Lancet D. GeneCards: encyclopaedia for genes, proteins and diseases. Trends Genet 1997; 13: 163.
22 Bustin SA, Jenkins PJ. The growth hormone-insulin-like growth factor-I axis and colorectal cancer. Trends Mol Med 2001; 7: 447-454.

23 Matzinger P. An innate sense of danger. Semin Immunol 1998; 10: 399-415.

24 Conley ME, Delacroix DL. Intravascular and mucosal immunoglobulin A: two separate but related systems of immune defense. Ann Intern Med 1987; 106: 892-899.

25 Mäkelä PH. Vaccines, coming of age after 200 years. FEMS Microbiol Rev 2000; 24: 9-20.

26 Krishnan S, Haensler J, Meulien P. Paving the way towards DNA vaccines. Nat Med 1995; 1: 521-522.

27 Roman $\mathrm{M}$ et al. Immunostimulatory DNA sequences function as T helper-1-promoting adjuvant. Nat Med 1997; 3: 849-854.

28 Quiding-Järbrink $\mathrm{M}$ et al. Differential expression of tissuespecific adhesion molecules on human circulating antibodyforming cells after systemic, enteric, and nasal immunizations. J Clin Invest 1997; 99: 1281-1286.

29 Surh CD, Sprent J. T-cell apoptosis detected in situ during positive and negative selection in the thymus. Nature 1994; 372: 100-103.

30 Hildeman DA et al. Molecular mechanisms of activated T-cell death in vivo. Curr Opin Immunol 2002; 14: 354-359.

31 Kiefer MC et al. Modulation of apoptosis by the widely distributed Bcl-2 homologue Bak. Nature 1995; 374: 736-739.

32 Boise LH et al. Bcl-x, a bcl-2-related gene that functions as a dominant regulator of apoptotic cell death. Cell 1993; 74: 597-608.

33 Wang $\mathrm{K}$ et al. Bcl-2 heterodimerizes in vivo with a conserved homolog, Bax, that accelerates programmed cell death. Cell 1993. 74: 609-619.

34 Crispe IN. Death and destruction of activated T lymphocytes. Immunol Res 1999; 19: 143-157.

35 Alderson MR et al. Fas ligand mediates activation-induced cell death in human T lymphocytes. J Exp Med 1995; 181: 71-77.

36 Vito P, Lacana E, D'Adamio L. Interfering with apoptosis: $\mathrm{Ca}(2+)$ binding protein ALG-2 and Alzheimer's disease gene ALG-3. Science 1996; 271: 521-525.

37 Mitchell TC et al. Immunological adjuvants promote activated T cell survival via induction of Bcl-3. Nat Immunol 2001; 13: 114-119.

38 Hamad AR, Schneck JP. Antigen-induced T-cell death is regulated by CD4 expression. Int Rev Immunol 2001; 20: 535-546.

39 Rebello A et al. Bcl-3 expression promotes cell survival following interleukin 4 deprivation and is controlled by AP1 and AP1 like transcription factors. Mol Cell Biol 2000; 20: 3407-3416.

40 Tavazoie S, Church GM. Quantitative whole-genome analysis of DNA-protein interactions by in vivo methylase protection in $E$. coli. Nat Biotechnol 1998; 16: 566-571.

41 Helander IM, Latva-Kala K, Lounatmaa K. Permeabilizing action of polyethyleneimin on Salmonella typhimurium involves disruption of the outer membrane with lipopolysaccharide. Microbiology 1998; 144: 385-390.

42 Helander IM, Alakomi HL, Latva-Kala K, Koski P. Polyethyleneimine is an effective permeabilizer of Gram-negative bacteria. Microbiology 1997; 143: 3193-3199.

43 Lashuel HA et al. Neurodegenerative disease: amyloid pores from pathogenic mutations. Nature 2002; 418: 291.

44 Godbey WT, Wu KK, Hirasaki GJ, Mikos AG. Improved packing of poly(ethylene-imine)/DNA complexes increases transfection efficiency. Gene Therapy 1999; 6: 1380-1388.

45 Eisen MB, Spellman PT, Brown PO, Botstein D. Cluster analysis and display of genome-wide expression patterns. Proc Natl Acad Sci USA 1998; 95: 14863-14868. 